\title{
ARTICLE Combat exposure, posttraumatic stress disorder, and head injuries differentially relate to alterations in cortical thickness in military Veterans
}

\author{
Ashley N. Clausen ${ }^{1,2}$, Emily Clarke $\mathbb{D}^{1,2}$, Rachel D. Phillips $\mathbb{i D}^{1,2}$, Courtney Haswell ${ }^{1,2}$, VA Mid-Atlantic MIRECC Workgroup ${ }^{1}$ and
} Rajendra A. Morey ${ }^{1,2,3,4}$

Combat-exposed Veterans are at increased risk for developing psychological distress, mood disorders, and trauma and stressorrelated disorders. Trauma and mood disorders have been linked to alterations in brain volume, function, and connectivity. However, far less is known about the effects of combat exposure on brain health. The present study examined the relationship between severity of combat exposure and cortical thickness. Post-9/11 Veterans ( $N=337 ; 80 \%$ male) were assessed with structural neuroimaging and clinically for combat exposure, depressive symptoms, prior head injury, and posttraumatic stress disorder (PTSD). Vertex-wide cortical thickness was estimated using FreeSurfer autosegmentation. FreeSurfer's Qdec was used to examine relationship between combat exposure, PTSD, and prior head injuries on cortical thickness (Monte Carlo corrected for multiple comparisons, vertex-wise cluster threshold of $1.3, p<0.01$ ). Covariates included age, sex, education, depressive symptoms, nonmilitary trauma, alcohol use, and prior head injury. Higher combat exposure uniquely related to lower cortical thickness in the left prefrontal lobe and increased cortical thickness in the left middle and inferior temporal lobe; whereas PTSD negatively related to cortical thickness in the right fusiform. Head injuries related to increased cortical thickness in the bilateral medial prefrontal cortex. Combat exposure uniquely contributes to lower cortical thickness in regions implicated in executive functioning, attention, and memory after accounting for the effects of PTSD and prior head injury. Our results highlight the importance of examining effects of stress and trauma exposure on neural health in addition to the circumscribed effects of specific syndromal pathology.

Neuropsychopharmacology (2020) 45:491-498; https://doi.org/10.1038/s41386-019-0539-9

\section{INTRODUCTION}

Over 2.7 million US service members have deployed as part of Operation Enduring (OEF), Iraqi Freedom (OIF), and New Dawn (OND; [1]). Military personnel serving since the onset of OEF may be subjected to multiple deployments, increasing exposure to combat trauma, and risk of combat-related disorders [2, 3]. Severe, prolonged stress associated with combat deployments has been consistently linked with increased prevalence of co-occurring psychological disorders, including PTSD, depression, alcohol misuse, and anxiety [3-8], and decreased executive function and sustained attention, and increased reaction time [9]. Psychological disorders relate to combat exposure and neuropsychological dysfunction [10-12], and may mediate the relationship between combat exposure and neuropsychological abilities. However, the results from one study suggest post-deployment neuropsychological performance remained significantly altered after controlling for effects of PTSD and depressive symptoms, suggesting a unique impact of combat exposure on brain function [9].

Mechanistic research linking chronic stress to changes in the brain highlights a critical role for the hypothalamic-pituitary-adrenal (HPA) axis [13-16]. In animal and human studies, chronic stress is associated with dysregulated cortisol levels, cytokines, glucocorticoid receptors, and corticotropin-releasing hormone levels, increased catecholamine levels, decreased acetylcholine, and increased pro-inflammatory response [13-16]. This dysregulation may weaken a cells' resilience, lead to cellular atrophy, and/or inhibited neurogenesis [13]. In Veterans, combat exposure and stress relate to lower grey matter volume within the left ventromedial prefrontal and bilateral dorsal anterior cingulate cortex [17], and lower grey matter volume in the right posterior insula [18].

Cortical thickness, another index of neural health, is a proxy for neuronal density and provides more specificity of brain-related changes as compared with grey matter volume [19]. Decreased cortical thickness is associated with decreased cognitive abilities in adults [20-22]. Thus, cortical thickness is a prime biomarker for our study to further interrogate established associations between combat exposure and alterations in cognitive abilities [9]. A small number of studies have examined differential effects of prolonged stress on cortical thickness in combat Veterans [23-26]. One study found Veterans with chronic pain exhibited an inverse relationship between combat and cortical thickness in the left inferior frontal gyrus, right precentral gyrus, and right superior parietal gyrus, and those without chronic plain displayed a positive relationship [25]. Similarly, Veterans exposed to childhood trauma exhibit a positive

\footnotetext{
'VA Mid-Atlantic MIRECC, Durham VAHCS, 508 Fulton St, Durham, NC 27705, USA; ${ }^{2}$ Duke-UNC Brain Imaging and Analysis Center, Duke University, Durham, NC, USA; ${ }^{3}$ Center for Cognitive Neuroscience, Duke University, Durham, NC, USA and ${ }^{4}$ Department of Psychiatry and Behavioral Sciences, Duke University, Durham, NC, USA Correspondence: Ashley N. Clausen (ashley.clausen@duke.edu)

Authors included in the VA Mid-Atlantic MIRECC Workgroup research group are listed in the Acknowledgements section.
}

Received: 20 May 2019 Revised: 23 September 2019 Accepted: 1 October 2019

Published online: 10 October 2019 
relationship between PTSD symptoms and cortical thickness within the paracentral and posterior cingulate, whereas Veterans without childhood trauma demonstrate a negative relationship [26]. This line of research suggests early life trauma and chronic pain influence combat-related changes in cortical thickness.

Research examining the unique effects of combat exposure in relation to cortical thickness indicates severity of combat exposure relates to lower cortical thickness within the left lateral prefrontal cortex, regardless of PTSD diagnosis [23, 24]. In addition, PTSD may moderate the relationship between combat stress and cortical thickness. Both studies investigating these intersecting relationships suggest stronger negative relationships between combat exposure and cortical thickness in Veterans without a PTSD diagnosis than Veterans with PTSD [23, 24]. These results suggest combat exposure may have a unique, negative impact on cortical thickness. However, the role of highly comorbid conditions, particularly head injury and depression, were not previously ascertained. This study sought to examine the role of highly comorbid conditions in combat Veterans in assessing the associations between combat and cortical thickness. If replicated, this line of research will underscore the importance of examining neural health in combat-exposed Veterans regardless of PTSD diagnosis.

Given the high prevalence rate of PTSD in OEF/OIF/OND Veterans [27], it is unsurprising most research has focused on the impact of PTSD on neural health as opposed to the effect of combat exposure. PTSD diagnosis and severity relate to lower cortical thickness primarily within the postcentral gyri, insular cortex, precuneus, fusiform, and entorhinal cortices, with more widespread findings (i.e., more regions throughout the brain in which PTSD related to lower cortical thickness) when examining lifetime burden of PTSD symptoms (cumulative effect of PTSD symptoms [28]). PTSD has also been linked with lower cortical thickness within the prefrontal cortex when controlling for depressive symptoms [23, 29]. PTSD-specific research provides significant insight into the effects of this particular diagnosis on neural health. However, diagnosis-specific approaches limit a critical understanding of how extended exposure to lifethreatening stressors, i.e., combat exposure, may impact neural health in all Veterans, not just those meeting diagnostic criteria for PTSD.

Another complicating factor of combat on neural health is head injury. Approximately $19 \%$ of military personnel sustain a head injury during a deployment, a significant risk factor for the development of PTSD, with the vast majority of head injuries being classified as mild traumatic brain injuries [30], and accelerated brain aging [31]. In Veterans, history of a head injury is associated with decreased cortical thickness within the right inferior temporal, right insular, and right inferior frontal gyrus [32]. Similarly, Veterans with both PTSD and head injury exhibit lower cortical thickness within the postcentral and middle temporal gyri [28]. However, it remains unclear how combat may affect cortical thickness beyond the effects of PTSD and previous head injuries.

This study examined the impact of combat on neural health as measured by cortical thickness. In addition, we examined whether the effects of combat are distinct from the influence of PTSD and previous head injuries in a large sample of OEF/OIF/OND Veterans. We adopted a data-driven approach to identify regions uniquely associated with combat exposure, while controlling for common combat-related conditions. Based on prior research examining the relationship between combat and cortical thickness [23, 24], we hypothesized severity of combat exposure would relate to lower cortical thickness in regions within the left frontal cortex independent of PTSD diagnosis. In addition, we hypothesized PTSD, above and beyond the effects of combat, would relate to decreased cortical thickness throughout the prefrontal cortex, and prior head injuries would relate to lower cortical thickness in the temporal lobes. To further explore intersecting relationships of combat, PTSD and head injuries, we also explored interaction effects of combat, PTSD, and head injuries on cortical thickness.

\section{MATERIALS AND METHODS}

Subjects

Subjects were recruited between 2005 and 2018 from a postdeployment mental health repository of 3877 US military Veterans [33]. Subjects included Veterans who served since 11 September 2001 between the ages of 18 and 59. Subjects underwent screening for inclusion and exclusion criteria based on information available in this repository and subsequent telephone contact. Full inclusion and exclusion criteria are included in Supplementary Material. Important exclusion criteria included penetrating head injury, loss of consciousness $\geq 30 \mathrm{~min}$, history of neurological disorders (e.g., seizure, stroke, multiple sclerosis, chronic encephalitis, cerebrovascular disease, CNS neoplasm), severe chronic medical conditions (e.g., liver failure, HIV, renal failure, heart failure, severe metabolic disturbance), neurosurgery, and Axis I psychiatric disorders other than PTSD and co-morbid Major Depression. A total of 341 Veterans met inclusion criteria for the present analysis. All subjects provided written informed consent. Study procedures were approved by the Institutional Review Boards at Duke University Medical Center and Durham VA Medical Center.

\section{Clinical assessment}

Clinical information was available from the post-deployment mental health repository. Diagnostic assessment of mental health was based in the DSM-IV-Text Revision [34], and assessed using the Structured Clinical Interview for DSM-IV Axis 1 Disorders (SCID; [35]). SCIDs were administered by trained research personnel. The Beck Depression Inventory (BDI-II; [36]), Combat Exposure Scale (CES; [37]), and the Alcohol Use Disorders Identification Test (AUDIT; [38]) were used to assess depression, severity of combat exposure, and alcohol use, respectively. The Traumatic Life Events Questionnaire (TLEQ) pre- and post-military index scores were summed to estimate non-military trauma [39]. Veterans selfreported history of head injuries by indicating (yes/no) if they had been diagnosed with a head injury on the physical health subscale of the National Vietnam Veterans' Readjustment Study Medical Questionnaire [40]. A supplemental analysis was conducted in a subset of Veterans $(n=205)$ who completed a traumatic brain injury interview ([33]; see Supplementary Material and Supplementary Fig. S2).

\section{Neuroimaging assessment}

Scanning was conducted on a GE Discover MR750 3.0 Tesla MRI scanner. A T1-weighted anatomical image was acquired using a 3D FSPGR sequence $\left(\mathrm{TR} / \mathrm{TE}=7.53 / 2.9 \mathrm{~ms}\right.$, flip $=12^{\circ}, \mathrm{FOV}=240$ $\mathrm{mm}, 256 \times 256 \times 162$ matrix, $1 \mathrm{~mm}$ slices). Images were preprocessed using FreeSurfer version 5.3.0 image analysis suite (http:// surfer.nmr.mgh.harvard.edu/ [41]). Quality assurance was conducted according to the ENIGMA Cortical Quality Control Guide 2.0 (available at http://enigma.ini.usc.edu/protocols/imagingprotocols/) by two trained raters. Four scans failed quality assurance due to pathology, leaving a total of 337 scans available for analysis. Cortical thickness estimates were extracted bilaterally from individual anatomical images using the ENIGMA cortical thickness pipeline (available at http://enigma.usc.edu).

\section{Statistical analyses}

Relationships between combat, PTSD, and cortical thickness were examined using FreeSurfer's Qdec (Monte Carlo corrected for multiple comparisons, vertex-wise cluster threshold of 1.3, $p<0.01$ [42]). Qdec is a vertex-based (whole-brain) general linear model approach developed to identify relationships between clusters of vertex-wise cortical thickness and a predictor variable (i.e., severity 
of combat exposure). This approach identifies regions of the cortex significantly related to the predictor and maps these clusters onto a predefined naming scheme based on the DesikanKilliany atlas [41]. We examined main and interaction effects of combat exposure, PTSD diagnosis, and head injuries adjusting for age, sex, education, prior head injury, depression, alcohol use, and nonmilitary trauma. Effect sizes were calculated for each effect. Subject-level mean cortical thickness values were extracted from each identified region of interest. Linear models were used to assess multiple adjusted $R^{2}$ values, and calculate Cohen's $f^{2}$. Education data were missing for one subject, and was imputed using the average score (3.9, equivalent to a technical trade school or an associate degree). Given the relationship between age and decreased cortical thickness [43], interaction effects of age were also examined (see Supplementary Material and Supplementary Fig. S3). All analyses were conducted using R Statistical Software Package (version 3.4.1; available at cran.org).

\section{RESULTS}

Demographic and clinical characteristics

Demographic and clinical characteristics are presented in Table 1 and Supplementary Table S1. In the present sample, $28.2 \%$ of Veterans met criteria for PTSD, and $10.7 \%$ of the sample endorsed experiencing at least one head injury. Severity of combat exposure ranged from 0 (light) to 37 (heavy), with an average severity of light to moderate (mean $=10.5, \mathrm{SD}=10.5$ ). There was a large range of depression symptoms from 0 (minimal) to 58 (severe), with average in the minimal range (mean $=10.8, \mathrm{SD}=11.6$ ). Average alcohol use was below the threshold for harmful drinking (threshold $\geq 8$; mean $=3.8, \mathrm{SD}=4.5$; range $0-26$ ). The number of noncombat trauma exposures ranged from 0 to 20 (mean $2.1, \mathrm{SD}=2.8$ ).

Vertex-wise analysis of cortical thickness

Qdec results, including peak z-values, peak coordinates, anatomical location, and effect sizes for each model, are presented in Table 2. Higher combat exposure related to lower cortical thickness in the left rostral middle frontal (peak $z=-3.32$ ) and right lateral orbitofrontal (peak $z=-3.35$ ) cortices (Supplementary Fig. S1). When controlling for the effects of PTSD, Qdec analysis revealed severity of combat exposure negatively relates to cortical thickness in regions within the left hemisphere spanning the medial prefrontal and superior frontal cortex (peak $z=-3.94$ ) and positively relates to cortical thickness in the lateral inferior and posterior cortex (peak $z=5.61$; Fig. 1a). PTSD, when controlling for combat exposure, significantly related to lower

Table 1. Demographics

\begin{tabular}{lll}
\hline & Mean & SD \\
\hline Age & 38.3 & 10.1 \\
Combat exposure scale total & 10.5 & 10.5 \\
BDI-II total severity score & 10.8 & 11.6 \\
AUDIT total severity score & 3.8 & 4.5 \\
TLEQ non-combat trauma & 2.1 & 2.8 \\
severity score & & \\
Education & 3.9 & 1.7 \\
& Endorsed (\%) & Endorsed $(N)$ \\
Sex (\% male) & $80.1 \%$ & 270 \\
Head injuries (\% with $\geq 1$ injury) & $10.7 \%$ & 36 \\
PTSD diagnosis (\% with PTSD) & $28.2 \%$ & 95 \\
\hline
\end{tabular}

BDI-II Beck Depression Inventory-II, AUDIT Alcohol Use Identification Test, TLEQ Traumatic Life Exposure Questionnaire, SD Standard Deviation, PTSD Posttraumatic Stress Disorder cortical thickness than non-PTSD controls within the right fusiform (peak $z=-3.07$ ), extending into the lateral occipital and inferior temporal cortex (Fig. 1b). An interaction effect was identified for combat exposure and PTSD on cortical thickness within the right precuneus (peak $z=5.74$ ) extending into the isthmus cingulate (Fig. 2). Veterans with PTSD exhibited a steeper, positive correlation between combat exposure and cortical thickness compared with Veterans without PTSD.

Veterans who endorsed a head injury exhibited increased cortical thickness within the bilateral medial prefrontal cortex (Fig. 3a). Veterans with a head injury exhibited increased cortical thickness within the left rostral anterior cingulate cortex (peak $z=$ 4.12) extending into the medial orbitofrontal, superior frontal, and caudal anterior cingulate cortices, the left rostral middle frontal cingulate (peak $z=3.36$ ) extending into the lateral orbitofrontal cortex, and the right rostral anterior cingulate (peak $z=2.87$ ) extending from the posterior cingulate to the medial orbitofrontal cortex. Veterans with and without head injuries exhibited an inverse relationship between combat exposure and cortical thickness within the right superior frontal cortex (peak $z=4.36$; Fig. 3b). Veterans who endorsed a head injury exhibited increased cortical thickness in this region compared with Veterans without head injuries. When examining PTSD diagnosis, Veterans with a head injury and PTSD diagnosis exhibited increased cortical thickness within the bilateral superior frontal cortex (right hemisphere peak $z=3.90$, left hemisphere peak $z=4.69$ ) compared with those without a PTSD diagnosis (Fig. 3c).

\section{DISCUSSION}

This study examined the impact of combat exposure on vertexwise cortical thickness in a large sample of OEF/OIF/OND combat Veterans. We specifically sought to clarify the unique contributions of combat exposure on cortical thickness by controlling for the influence of PTSD and sustained head injuries. Using a Qdec analysis, we identified (1) severity of combat exposure negatively related to cortical thickness in regions spanning the left superior prefrontal cortex and positively related to cortical thickness in the left inferior and posterior cortices; (2) PTSD diagnosis was negatively related to cortical thickness within the right fusiform; and (3) history of head injury related to increased cortical thickness bilaterally within the medial prefrontal cortex. The lateralization and directionality of results points to a differential impact of combat, PTSD, and head injuries on neural health.

Prior research examining combat and PTSD conducted in small samples of OEF/OIF/OND Veterans with $N=69$ [23] and $N=41$ [24], provides evidence that combat exposure relates to alterations in cortical thickness. Furthermore, while these previous investigations included adjustments for prior head injuries, neither explored the unique effects of head injuries on cortical thickness, representing a critical gap in our current understanding of how combat exposure may uniquely impact cortical thickness. This study explored relationships between severity of combat exposure, PTSD, and head injuries with cortical thickness in a sample of 337 Veterans. In this comparatively large sample, we identified small to medium effects of combat, PTSD, and prior head injuries on cortical thickness with Cohen's $f^{2}$ ranging from 0.09 to 0.32 (Table 2). Power analyses based on the present results (Cohen's $f^{2}$ $=0.09$ and $0.32, a=0.05,1-\beta=0.8$, three tested predictors [combat, PTSD and head injuries], eight total [controlling for age, sex, education, depression, and alcohol use]) indicate minimum required sample sizes ranging from 56 to 175 , suggesting previous research may have been underpowered to detect true main effect relationships with cortical thickness [44]. Present findings largely confirm previously underpowered studies, providing additional support for a significant relationship between combat exposure and lower cortical thickness beyond the effects of PTSD and previously sustained head injuries. 
Table 2. Qdec results for the effects of combat, PTSD, and head injuries on cortical thickness

\begin{tabular}{|c|c|c|c|c|c|c|}
\hline Cluster & Peak $z$-value & Cluster size $\left(\mathrm{mm}^{2}\right)$ & $x, y, z$ coordinates & Adjusted $R^{2}$ & Cohen's $f 2$ & Region of peak $z$-value \\
\hline 1 & -3.32 & 2086.15 & $-28.9,52.2,-6.2$ & 0.19 & 0.23 & Left rostral middle frontal cortex \\
\hline \multicolumn{7}{|c|}{ Combat exposure controlling for PTSD } \\
\hline 1 & 5.61 & 8088.91 & $-43.4,-49.7,12.5$ & 0.22 & 0.28 & Left banks superior temporal sulcus \\
\hline 2 & -3.94 & 2825.25 & $-13.4,35.5,18.6$ & 0.24 & 0.32 & Left superior frontal \\
\hline 1 & -3.07 & 2086.81 & $40.4,-56.4,-15.1$ & 0.08 & 0.09 & Right fusiform \\
\hline \multicolumn{7}{|c|}{ Interaction of combat severity and PTSD } \\
\hline 1 & 5.74 & 1378.77 & $14.2,-58.3,23.5$ & 0.15 & 0.18 & Right precuneus \\
\hline \multicolumn{7}{|c|}{ Head injury controlling for combat exposure and PTSD } \\
\hline 1 & 4.12 & 2549.48 & $-10.7,33.2,18.2$ & 0.20 & 0.25 & Left rostral anterior cingulate cortex \\
\hline \multicolumn{7}{|c|}{ Interaction of head injury and PTSD } \\
\hline 1 & 3.90 & 1553.01 & $-8.9,14.3,54.7$ & 0.21 & 0.27 & Left superior frontal cortex \\
\hline 2 & 4.69 & 1769.85 & $8.5,62.5,-0.5$ & 0.10 & 0.11 & Right superior frontal cortex \\
\hline
\end{tabular}

Consistent with previous findings, present results indicate severity of combat exposure significantly relates to lower cortical thickness within the left medial prefrontal and superior frontal cortex, including rostral middle frontal, superior frontal, medial orbitofrontal, and rostral anterior cingulate cortices when controlling for depressive symptom severity, history of head injury, nonmilitary trauma, alcohol use, age, sex, and education (Fig. 1a). The medial and superior aspects of the prefrontal cortex have been consistently implicated in a range of cognitive functions, including executive functioning, decision-making, reward processing, sustained attention, memory, and emotion processing and regulation [45]. In fact, we have previously shown a relationship between increased PTSD severity in Veterans and increased functional activation during emotionally valenced stimuli (compared with neutral stimuli) in the ventral frontolimbic regions, and decreased activation during executive function tasks within the middle frontal gyrus, dorsal anterior cingulate, and inferior parietal lobule [46]. In addition, another study examining the relationship between cortical thickness and cognitive inhibition indicates Veterans with PTSD-related disinhibition exhibited lower cortical thickness within the left medial orbitofrontal, rostral anterior cingulate, and frontal pole [47]. However, these previous studies did not examine the influence combat exposure may have on cortical thickness or brain function. Therefore, current findings suggest alterations within the frontal lobe may be a result of combat exposure severity as opposed to being solely circumscribed to PTSD diagnosis.

We also identified a positive relationship between combat exposure and cortical thickness in the left middle and inferior temporal cortex (Fig. 1b), extending into the fusiform, lateral occipital, banks of the superior temporal sulcus, inferior parietal, and supramarginal cortices. Acute onset of PTSD has been significantly associated with reduced cortical thickness in bilateral frontal and inferior parietal regions, and right lateral occipital lobe [48]. The lateral occipital cortex is thought to play a role in the integration of multisensory information and object recognition $[49,50]$. While functional implications of reduced cortical thickness in relation to PTSD diagnosis are speculative given our current focus on structural alterations, our findings are consistent with the dysfunction in visual and multisensory processing and recognition reported in combat-exposed Veterans. In fact, previous research in Veterans, suggests PTSD is linked with reduced functional activation of the lateral occipital lobe during repeated exposure to emotionally valenced stimuli, suggesting this region may underlie difficulties with habituation in Veterans with PTSD [51]. Notably, previous research did not control for trauma exposure. Thus, our results indicate combat exposure may have a unique impact on cortical thickness within the lateral middle and inferior temporal regions.

To determine if the observed pattern of cortical thickness was unique to combat severity, we also explored the relationship between PTSD and cortical thickness, controlling for combat exposure. Our results were partially consistent with previous findings in Veterans which identify a unique impact of combat and PTSD on cortical thickness. However, prior findings identified more widespread effects of PTSD on cortical thickness, particularly lower cortical thickness throughout the frontal cortex [23]. In comparison, our results suggest PTSD may have a unique impact on cortical thickness within the right fusiform (Fig. 1c), which parallels findings associated with PTSD and grey matter volume in this region [52]. More specifically, Veterans with a diagnosis of PTSD exhibited lower cortical thickness within the right fusiform cortex extending into the lateral occipital, and inferior temporal cortices. This region is associated with pattern, object, and facial recognition. We have previously found Veterans with PTSD, compared to trauma-exposed controls, exhibit increased activation of this region when viewing emotional, trauma-related cues [53]. Thus, present results provide additional evidence linking PTSD with alterations in the fusiform region.

In line with previous research conducted in smaller samples $[23,24]$, we found an interaction effect of PTSD on the relationship between combat exposure and cortical thickness. Veterans with PTSD exhibited a steeper positive relationship between combat exposure and cortical thickness compared with Veterans without 
A. Effects of combat on cortical thickness controlling for PTSD
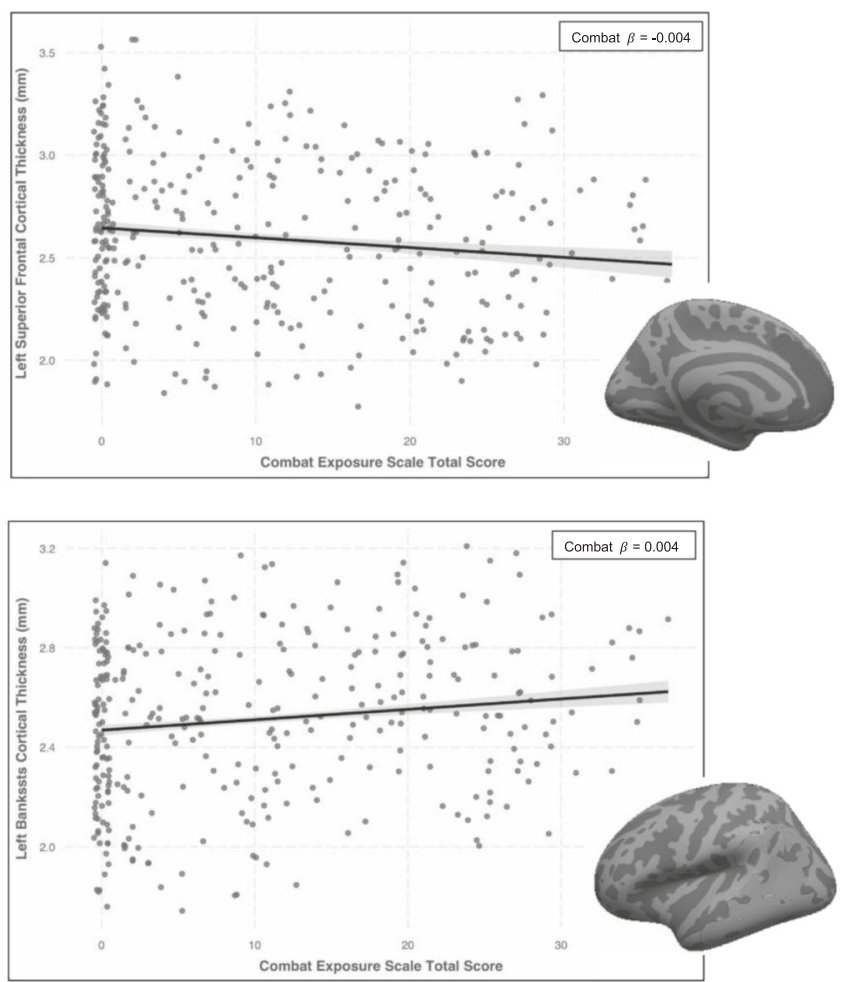

B. Effects of PTSD on cortical thickness controlling for combat

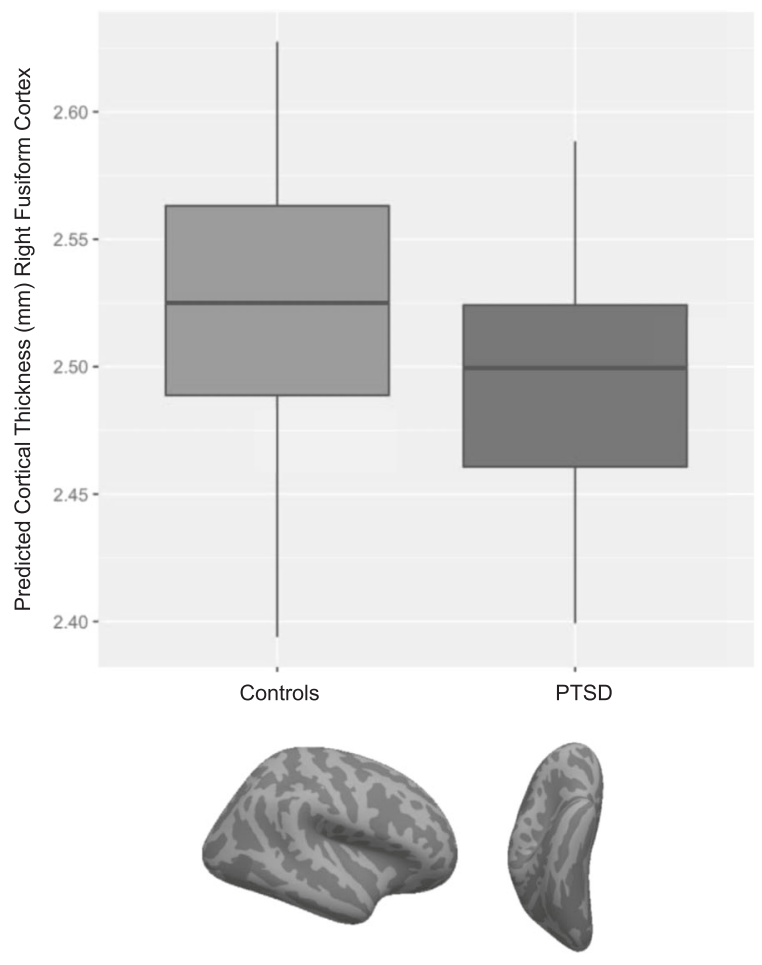

Fig. 1 Relationship between combat exposure, PTSD, and cortical thickness. a Combat severity, controlling for PTSD, related to alterations in cortical thickness in the left superior frontal cortex, including increased cortical thickness in the left banks of the superior temporal sulcus (peak $z=5.61$, peak $x, y, z$ coordinates $=-43.4,-49.7,12.5$, model $R^{2}=0.22$, combat severity $\beta=0.004$ ), and lower cortical thickness in the left superior frontal cortex (peak $z=-3.94$, peak $x, y, z$ coordinates $=-13.4,35.5,18.6$, model $R^{2}=0.24$, combat severity $\beta=-0.004$ ). b PTSD diagnosis, controlling for combat exposure, relates to lower cortical thickness in the right fusiform (peak $z=-3.07$, peak $x, y, z$ coordinates $=$ $40.4,-56.4,-15.1$, model $R^{2}=0.08$, PTSD diagnosis $\beta=-0.03$ )

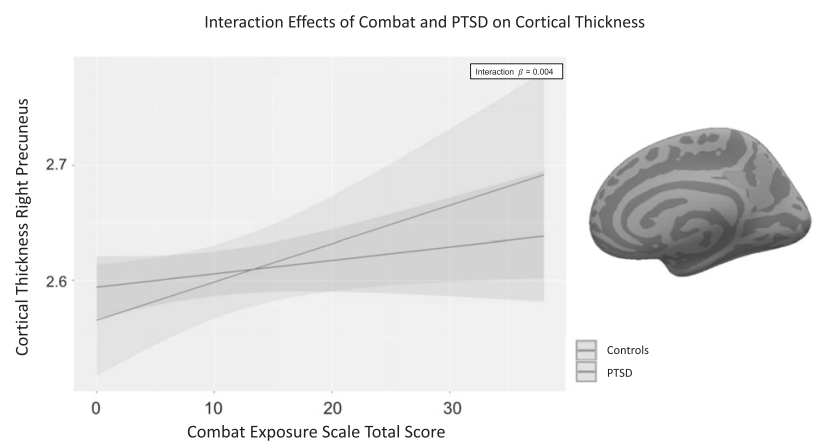

Fig. 2 Interaction of combat and PTSD on cortical thickness. Veterans with PTSD exhibited a positive relationship between combat exposure and cortical thickness in the right hemisphere; whereas those without PTSD exhibited a weaker relationship within the right precuneus (peak $z=5.74$, peak $x, y, z$ coordinates $=14.2$, $-58.3,23.5$, model $R^{2}=0.15$, interaction $\beta=0.004$

PTSD in the right precuneus (Fig. 2), extending into the isthmus cingulate. The precuneus is implicated in visuospatial imagery, episodic memory, and self-referential processing [54]. Our results are in line with functional neuroimaging research, suggesting PTSD is associated with hyperactivation of this region during symptom provocation [55]. Unexpectedly, Veterans with PTSD exhibited lower cortical thickness at lower levels of combat exposure compared with those without PTSD, and those with PTSD exhibited a positive relationship between combat severity and cortical thickness in these regions. Therefore, it is possible lower cortical thickness prior to combat exposure may represent a risk factor for the development of PTSD. However, longitudinal research is needed to further understand the temporal relationships between combat exposure, development of PTSD, and cortical thickness.

We also examined the impact of head injuries on cortical thickness controlling for both combat exposure and PTSD. In the context of Veteran populations, one study examining the effects of mild head injuries on cortical thickness in a small sample of Veterans found mild head injuries were associated with decreased cortical thickness within the right inferior temporal, insular, and inferior frontal gyrus [32]. Similarly, Veterans with both a history of PTSD and a head injury exhibit lower cortical thickness within the postcentral and middle temporal gyri [28]. In contrast to previous findings, prior diagnosis of a head injury in this study significantly related to increased cortical thickness within bilateral rostral anterior cingulate and left rostral middle frontal cortices (Fig. 3a). As noted above, regions in the medial frontal cortex are involved in higher order functions, including executive functioning, decision-making, and emotional processing [45]. A similar pattern of cortical thickness emerged when examining the interaction of the combat exposure severity (Fig. 3b) and PTSD (Fig. 3c) with head injuries on cortical thickness in the superior frontal cortex, in which Veterans with head injuries plus higher levels of combat or PTSD exhibited increased cortical thickness in this region. It is plausible increased cortical thickness may be associated with changes in functional and structural connectivity in Veterans who have sustained a head injury when controlling for the effects of PTSD and combat. Previous research in patients with and without head injuries suggests increased functional and structural connectivity with the default mode network, which encompasses 



C. Interaction of head injuries and PTSD on cortical thickness


Fig. 3 Main and interaction effects of prior head injuries and cortical thickness. a When controlling for combat exposure, and PTSD, prior head injuries relate to increased cortical thickness in the bilateral rostral anterior cingulate cortex (left hemisphere peak $z=4.12$, peak $x, y, z$ coordinates $=-10.7,33.2,18.2$, model $R^{2}=0.20$, head injury $\beta=0.05$; right hemisphere peak $z=2.87$, peak $x, y, z$ coordinates $=8.6,32.0,-8.6$, model $R^{2}=0.08$, head injury $\beta=0.04$ ), and the left rostral middle frontal cortex (peak $z=3.36$, peak $x, y, z$ coordinates $=-22.6,50.8,0.4$, model $R^{2}=0.12$, head injury $\beta=0.07$ ) when compared with those did not sustain a prior head injury. $\mathbf{b}$ Veterans with and without a head injury exhibited a negative relationship between combat exposure and cortical thickness within the right superior frontal cortex; however, those with a head injury exhibited increased cortical thickness in this region compared with those without a head injury (peak $z=4.36$, peak $x$, $y, z$ coordinates $=10.3,60.4,2.5$; model $R^{2}=0.11$; interaction $\left.\beta=0.001\right)$. c Veterans with a head injury and PTSD diagnosis exhibited increased cortical thickness compared with those with a head injury and no PTSD in the left (peak $z=3.90$, peak $x, y, z$ coordinates $=10.3,60.4,2.5$; model $R^{2}=0.21$; interaction $\beta=0.03$ ) and right (peak $z=4.69$, peak $x, y, z$ coordinates $=8.5,62.5,-0.5 ;$ model $R^{2}=0.10$; interaction $\beta=0.02$ ) superior frontal cortex

the prefrontal cortex, may represent a compensatory mechanism in patients with head injuries $[56,57]$. Furthermore, head injuries may also impact the functional and structural connectivity between networks leading to widespread network dysregulation [56-58]. However, the functional implications of increased cortical thickness are purely speculative, given our focus on structural assessment of neural health. Future research employing more thorough assessments of head injuries, and functional neuroimaging will help clarify if increased cortical thickness is in fact associated with compensatory processing in Veterans who have sustained head injuries.

\section{Limitations}

While present results suggest combat has a differential impact on neural health compared with the effects of PTSD, the study was not without limitations. First, head injuries were examined using a dichotomous self-report measure limiting our ability to examine the influence of number or severity of head injuries on cortical thickness, and limiting our ability to examine the impact of blast exposure with and without concussive symptoms. The number that endorsed head injuries in the present sample (10\%) is lower than prevalence rates of $20 \%$ in OEF/OIF/OND Veterans [30]. This discrepancy may be due to our assessment of diagnosed head injuries. Future research should include thorough assessment of head injuries and blast exposure. In addition, as with most studies examining combat-related PTSD, there were high levels of collinearity between combat severity, PTSD, depression, and TBI in the present sample (correlations ranging from 0.29 to 0.42 , all $\left.p^{\prime} s<0.01\right)$. This may influence our ability to determine the unique effects of combat, PTSD, and head injuries on cortical thickness. Assessment of depression, combat, alcohol use, head injury, and trauma exposure were based on self-report measures, which are subject to bias. The SCID was used to assess PTSD. While the SCID is a validated assessment of PTSD diagnosis, the gold-standard assessment is the Clinician Administered PTSD Scale (CAPS; [59-61]), which was only available in 79\% of the sample. Last, many individuals with PTSD are prescribed psychotropic medication, which may impact neural health [62]. Medication information was not assessed in the full sample warranting future research to examining potential effects of psychotropic medication on cortical thickness.

\section{CONCLUSION}

We examined the impact of combat exposure on neural health by assessing the contributions of various sources of heterogeneity in a large sample of combat Veterans $(N=337)$. The results show combat exposure severity, controlling for PTSD, significantly relates to lower cortical thickness within the left prefrontal lobe, including the rostral middle frontal, superior frontal, medial orbitofrontal, and rostral anterior cingulate cortices, and increased cortical thickness within the left middle and inferior temporal cortex. PTSD relates to lower cortical thickness within the right fusiform extending into the lateral occipital and inferior temporal cortex. Veterans who sustained a head injury exhibit increased cortical thickness bilaterally in the medial prefrontal cortex. This 
may indicate combat exposure severity, PTSD, and prior head injuries have differential impacts on cortical thickness in Veteran populations. Utilization of longitudinal research designs and implementation of multimodal neuroimaging approaches (i.e., functional and structural assessments) are warranted and will clarify the functional implications of the present findings.

\section{FUNDING AND DISCLOSURE}

This research was supported by the U.S. Department of Veterans Affairs (VA) Mid-Atlantic Mental Illness Research, Education, and Clinical Center (MIRECC) core funds of the Department of Veterans Affairs Office of Mental Health Services. Dr. Morey also received financial support from the VA Office of Research and Development (5I01CX000748-01, 5I01CX000120-02). Additional financial support was provided by the National Institute for Neurological Disorders and Stroke (R01NS086885-01A1). Writing of this paper was supported by the Department of Veterans Affairs Office of Academic Affiliations Advanced Fellowship Program in Mental Illness Research and Treatment, the Medical Research Service of the Durham VA Health Care System, and the Department of Veterans Affairs Mid-Atlantic MIRECC. The views expressed in this article are those of the authors and do not necessarily reflect the position or policy of the Department of Veterans Affairs or the United States Government. The authors have no conflicts of interest to disclose, including the contributing members of the VA Mid-Atlantic MIRECC Workgroup.

\section{DATA AVAILABILITY}

The data that support the findings of this study are available on request from the corresponding author. The data are not publicly available due to privacy or ethical restrictions.

\section{ACKNOWLEDGEMENTS}

The VA Mid-Atlantic MIRECC Workgroup contributors for this paper include: Mira Brancu, PhD, Jean C. Beckham, PhD, Patrick S. Calhoun, PhD, Eric Dedert, PhD, Eric B. Elbogen, PhD, John A. Fairbank, PhD, Robin A. Hurley, MD, Jason D. Kilts, PhD, Nathan A. Kimbrel, PhD, Angela Kirby, MS, Christine E. Marx, MD, MS, Scott D. McDonald, PhD, Scott D. Moore, MD, PhD, Rajendra A. Morey, MD, MS, Jennifer C. Naylor, PhD, Jared Rowland, PhD, Cindy Swinkels, PhD, Steven T. Szabo, MD, PhD, Katherine H. Taber, PhD., Larry A. Tupler, PhD, Elizabeth E. Van Voorhees, PhD, H. Ryan Wagner, PhD, and Ruth E. Yoash-Gantz, PsyD.

\section{ADDITIONAL INFORMATION}

Supplementary Information accompanies this paper at (https://doi.org/10.1038/ s41386-019-0539-9)

Publisher's note Springer Nature remains neutral with regard to jurisdictional claims in published maps and institutional affiliations.

\section{REFERENCES}

1. Wenger JW, O'Connell C, Cottrell L. Examination of Recent Deployment Experience Across the Services and Components: Rand Arroyo Center Santa Monica CA Santa Monica US. Contract No.: RR-1928-A 2018.

2. Kline A, Falca-Dodson M, Sussner B, Ciccone DS, Chandler $\mathrm{H}$, Callahan $\mathrm{L}$, et al. Effects of repeated deployment to Iraq and Afghanistan on the health of New Jersey Army National Guard troops: implications for military readiness. Am J Public Health. 2010;100:276-83. https://doi.org/10.2105/AJPH.2009.162925.

3. Hoge CW, Castro CA, Messer SC, McGurk D, Cotting DI, Koffman RL. Combat duty in Iraq and Afghanistan, mental health problems, and barriers to care. N Engl J Med. 2004;351:13-22. https://doi.org/10.1056/NEJMoa040603.

4. Ginzburg K, Ein-Dor T, Solomon Z. Comorbidity of posttraumatic stress disorder, anxiety and depression: a 20-year longitudinal study of war veterans. J Affect Disord. 2010;123:249-57. https://doi.org/10.1016/j.jad.2009.08.006.

5. Hahn AM, Tirabassi CK, Simons RM, Simons JS. Military sexual trauma, combat exposure, and negative urgency as independent predictors of PTSD and subsequent alcohol problems among OEF/OIF veterans. Psychol Serv. 2015;12:378-83. https://doi.org/10.1037/ser0000060.

6. Campbell DG, Felker BL, Liu CF, Yano EM, Kirchner JE, Chan D, et al. Prevalence of depression-PTSD comorbidity: implications for clinical practice guidelines and primary care-based interventions. J Gen Intern Med. 2007;22:711-8. https://doi. org/10.1007/s11606-006-0101-4.

7. Ikin JF, Creamer MC, Sim MR, McKenzie DP. Comorbidity of PTSD and depression in Korean War veterans: prevalence, predictors, and impairment. J Affect Disord. 2010;125:279-86. https://doi.org/10.1016/j.jad.2009.12.005.

8. Ferrier-Auerbach AG, Erbes CR, Polusny MA, Rath CM, Sponheim SR. Predictors of emotional distress reported by soldiers in the combat zone. J Psychiatr Res. 2010;44:470-6. https://doi.org/10.1016/j.jpsychires.2009.10.010.

9. Vasterling JJ, Proctor SP, Amoroso P, Kane R, Heeren T, White RF. Neuropsychological outcomes of army personnel following deployment to the Iraq war. JAMA. 2006;296:519-29. https://doi.org/10.1001/jama.296.5.519.

10. Aupperle RL, Melrose AJ, Stein MB, Paulus MP. Executive function and PTSD: disengaging from trauma. Neuropharmacology. 2012;62:686-94. https://doi.org/ 10.1016/j.neuropharm.2011.02.008.

11. Dolan S, Martindale S, Robinson J, Kimbrel NA, Meyer EC, Kruse MI, et al. Neuropsychological sequelae of PTSD and TBI following war deployment among OEF/OIF veterans. Neuropsychol Rev. 2012;22:21-34. https://doi.org/10.1007/ s11065-012-9190-5.

12. Riley E, Mitko A, Stumps A, Robinson M, Milberg W, McGlinchey R, et al. Clinically significant cognitive dysfunction in OEF/OIF/OND veterans: prevalence and clinical associations. Neuropsychology. 2019;33:534-46. https://doi.org/10.1037/ neu0000529.

13. Frodl T, O'Keane V. How does the brain deal with cumulative stress? A review with focus on developmental stress, HPA axis function and hippocampal structure in humans. Neurobiol Dis. 2013;52:24-37. https://doi.org/10.1016/j. nbd.2012.03.012.

14. de Kloet ER, Joels $M$, Holsboer F. Stress and the brain: from adaptation to disease. Nat Rev Neurosci. 2005;6:463-75. https://doi.org/10.1038/nrn1683.

15. Kim YK, Won E. The influence of stress on neuroinflammation and alterations in brain structure and function in major depressive disorder. Behav Brain Res. 2017;329:6-11. https://doi.org/10.1016/j.bbr.2017.04.020.

16. Popoli M, Yan Z, McEwen BS, Sanacora G. The stressed synapse: the impact of stress and glucocorticoids on glutamate transmission. Nat Rev Neurosci. 2011;13:22-37. https://doi.org/10.1038/nrn3138.

17. Butler $\mathrm{O}$, Adolf J, Gleich T, Willmund G, Zimmermann $\mathrm{P}$, Lindenberger $\mathrm{U}$, et al. Military deployment correlates with smaller prefrontal gray matter volume and psychological symptoms in a subclinical population. Transl Psychiatry. 2017;7: e1031 https://doi.org/10.1038/tp.2016.288.

18. Clausen AN, Billinger SA, Sisante JV, Suzuki H, Aupperle RL. Preliminary evidence for the impact of combat experiences on gray matter volume of the posterior insula. Front Psychol. 2017;8:2151 https://doi.org/10.3389/fpsyg.2017.02151.

19. Winkler AM, Kochunov P, Blangero J, Almasy L, Zilles K, Fox PT, et al. Cortical thickness or grey matter volume? The importance of selecting the phenotype for imaging genetics studies. Neuroimage. 2010;53:1135-46. https://doi.org/10.1016/ j.neuroimage.2009.12.028.

20. McConathy J, Sheline YI. Imaging biomarkers associated with cognitive decline: a review. Biol Psychiatry. 2015;77:685-92. https://doi.org/10.1016/j. biopsych.2014.08.024.

21. Pacheco J, Goh JO, Kraut MA, Ferrucci L, Resnick SM. Greater cortical thinning in normal older adults predicts later cognitive impairment. Neurobiol Aging 2015;36:903-8. https://doi.org/10.1016/j.neurobiolaging.2014.08.031.

22. Cheng CP, Cheng ST, Tam CW, Chan WC, Chu WC, Lam LC. Relationship between cortical thickness and neuropsychological performance in normal older adults and those with mild cognitive impairment. Aging Dis. 2018;9:1020-30. https:// doi.org/10.14336/AD.2018.0125.

23. Wrocklage KM, Averill LA, Cobb Scott J, Averill CL, Schweinsburg B, Trejo M, et al. Cortical thickness reduction in combat exposed U.S. veterans with and without PTSD. Eur Neuropsychopharmacol. 2017;27:515-25. https://doi.org/10.1016/j. euroneuro.2017.02.010.

24. Averill LA, Abdallah CG, Pietrzak RH, Averill CL, Southwick SM, Krystal JH, et al. Combat exposure severity is associated with reduced cortical thickness in combat veterans: a preliminary report. Chronic Stress. 2017;1. https://doi.org/10.1177/ 2470547017724714

25. Corbo V, Salat DH, Powell MA, Milberg WP, McGlinchey RE. Combat exposure is associated with cortical thickness in Veterans with a history of chronic pain. Psychiatry Res Neuroimaging. 2016;249:38-44. https://doi.org/10.1016/j. pscychresns.2016.02.003.

26. Corbo V, Salat DH, Amick MM, Leritz EC, Milberg WP, McGlinchey RE. Reduced cortical thickness in veterans exposed to early life trauma. Psychiatry Res. 2014;223:53-60. https://doi.org/10.1016/j.pscychresns.2014.04.013. 
27. Fulton JJ, Calhoun PS, Wagner HR, Schry AR, Hair LP, Feeling N, et al. The prevalence of posttraumatic stress disorder in Operation Enduring Freedom/ Operation Iraqi Freedom (OEF/OIF) Veterans: a meta-analysis. J Anxiety Disord. 2015;31:98-107. https://doi.org/10.1016/j.janxdis.2015.02.003.

28. Lindemer ER, Salat DH, Leritz EC, McGlinchey RE, Milberg WP. Reduced cortical thickness with increased lifetime burden of PTSD in OEF/OIF Veterans and the impact of comorbid TBI. Neuroimage Clin. 2013;2:601-11. https://doi.org/ 10.1016/j.nicl.2013.04.009.

29. Geuze E, Westenberg HG, Heinecke A, de Kloet CS, Goebel R, Vermetten E. Thinner prefrontal cortex in veterans with posttraumatic stress disorder. Neuroimage. 2008;41:675-81. https://doi.org/10.1016/j.neuroimage.2008.03.007.

30. Stein MB, Kessler RC, Heeringa SG, Jain S, Campbell-Sills L, Colpe LJ, et al. Prospective longitudinal evaluation of the effect of deployment-acquired traumatic brain injury on posttraumatic stress and related disorders: results from the Army Study to Assess Risk and Resilience in Servicemembers (Army STARRS). Am J Psychiatry. 2015;172:1101-11. https://doi.org/10.1176/appi.ajp.2015.14121572.

31. Savjani RR, Taylor BA, Acion L, Wilde EA, Jorge RE. Accelerated changes in cortical thickness measurements with age in military service members with traumatic brain injury. J Neurotrauma. 2017;34:3107-16. https://doi.org/10.1089/neu.2017.5022.

32. Michael AP, Stout J, Roskos PT, Bolzenius J, Gfeller J, Mogul D, et al. Evaluation of cortical thickness after traumatic brain injury in military veterans. J Neurotrauma. 2015;32:1751-8. https://doi.org/10.1089/neu.2015.3918.

33. Brancu M, Wagner HR, Morey RA, Beckham JC, Calhoun PS, Tupler LA, et al. The post-deployment mental health (PDMH) study and repository: a multi-site study of US Afghanistan and Iraq era veterans. Int J Methods Psychiatr Res. 2017;26. https://doi.org/10.1002/mpr.1570.

34. American Psychiatric Association. Diagnostic and statistical manual-IV text revisions. Washington D.C.: American Psychiatric Association; 2000.

35. First MB, Spitzer RL, Gibbon M, Williams JB. Structured clinical interview for DSMIV axis I disorders, clinician version (SCID-CV). Washington D.C: American Psychiatric Press, Inc; 1996.

36. Beck AT, Steer RA, Brown GK. Beck depression inventory-II. Psychological Corporation. San Antonio: 1996.

37. Keane T, Fairbank JA, Caddell JM, Zimering RT, Taylor KL, Mora C. Clinical evaluation of a measure to assess combat exposure. Psychological Assess. 1989;1:53-5. https://doi.org/10.1037/1040-3590.1.1.53.

38. Saunders JB, Aasland OG, Babor TF, de la Fuente JR, Grant M. Development of the alcohol use disorders identification test (AUDIT): WHO collaborative project on early detection of persons with harmful alcohol consumption-Il. Addiction. 1993;88:791-804.

39. Kubany ES, Haynes SN, Leisen MB, Owens JA, Kaplan AS, Watson SB, et al. Development and preliminary validation of a brief broad-spectrum measure of trauma exposure: the Traumatic Life Events Questionnaire. Psychol Assess. 2000;12:210-24.

40. Kulka RA, Schlenger WE, Fairbank JA, Hough RL, Jordan BK, Marmar CR, et al. Contractual report of findings from the national vietnam veterans' readjustment study. Research Triangle Park, NC: Research Triangle Institute; 1988.

41. Fischl B. FreeSurfer. Neuroimage. 2012;62:774-81. https://doi.org/10.1016/j. neuroimage.2012.01.021

42. Hagler DJ Jr., Saygin AP, Sereno MI. Smoothing and cluster thresholding for cortical surface-based group analysis of fMRI data. Neuroimage 2006;33:1093-103. https://doi.org/10.1016/j.neuroimage.2006.07.036.

43. Lemaitre H, Goldman AL, Sambataro F, Verchinski BA, Meyer-Lindenberg A, Weinberger DR, et al. Normal age-related brain morphometric changes: nonuniformity across cortical thickness, surface area and gray matter volume? Neurobiol Aging. 2012;33:617 e1-9. https://doi.org/10.1016/j.neurobiolaging.2010.07.013.

44. loannidis JP. Why most published research findings are false. PLoS Med. 2005;2: e124 https://doi.org/10.1371/journal.pmed.0020124.
45. Seeley WW, Menon V, Schatzberg AF, Keller J, Glover GH, Kenna H, et al. Dissociable intrinsic connectivity networks for salience processing and executive control. J Neurosci. 2007;27:2349-56. https://doi.org/10.1523/JNEUROSCI.558706.2007.

46. Morey RA, Petty CM, Cooper DA, Labar KS, McCarthy G. Neural systems for executive and emotional processing are modulated by symptoms of posttraumatic stress disorder in Iraq War veterans. Psychiatry Res. 2008;162:59-72. https:// doi.org/10.1016/j.pscychresns.2007.07.007.

47. Sadeh N, Spielberg JM, Miller MW, Milberg WP, Salat DH, Amick MM, et al. Neurobiological indicators of disinhibition in posttraumatic stress disorder. Hum Brain Mapp. 2015;36:3076-86. https://doi.org/10.1002/hbm.22829.

48. Qi S, Mu YF, Cui LB, Zhang J, Guo F, Tan QR, et al. Anomalous gray matter structural networks in recent onset post-traumatic stress disorder. Brain Imaging Behav. 2018;12:390-401. https://doi.org/10.1007/s11682-017-9693-z.

49. Beauchamp MS. See me, hear me, touch me: multisensory integration in lateral occipital-temporal cortex. Curr Opin Neurobiol. 2005;15:145-53. https://doi.org/ 10.1016/j.conb.2005.03.011.

50. Amedi A, Jacobson G, Hendler T, Malach R, Zohary E. Convergence of visual and tactile shape processing in the human lateral occipital complex. Cereb Cortex. 2002;12:1202-12.

51. Hendler T, Rotshtein P, Hadar U. Emotion-perception interplay in the visual cortex: "the eyes follow the heart". Cell Mol Neurobiol. 2001;21:733-52.

52. Chao LL, Lenoci M, Neylan TC. Effects of post-traumatic stress disorder on occipital lobe function and structure. Neuroreport. 2012;23:412-9. https://doi.org/ 10.1097/WNR.0b013e328352025e.

53. Morey RA, Dolcos F, Petty CM, Cooper DA, Hayes JP, LaBar KS, et al. The role of trauma-related distractors on neural systems for working memory and emotion processing in posttraumatic stress disorder. J Psychiatr Res. 2009;43:809-17. https://doi.org/10.1016/j.jpsychires.2008.10.014.

54. Cavanna $A E$, Trimble MR. The precuneus: a review of its functional anatomy and behavioural correlates. Brain. 2006;129:564-83. https://doi.org/10.1093/brain/ awl004.

55. Sartory G, Cwik J, Knuppertz H, Schurholt B, Lebens M, Seitz RJ, et al. In search of the trauma memory: a meta-analysis of functional neuroimaging studies of symptom provocation in posttraumatic stress disorder (PTSD). PLoS One. 2013;8: e58150 https://doi.org/10.1371/journal.pone.0058150.

56. Sharp DJ, Beckmann CF, Greenwood R, Kinnunen KM, Bonnelle V, De Boissezon X, et al. Default mode network functional and structural connectivity after traumatic brain injury. Brain. 2011;134:2233-47. https://doi.org/10.1093/brain/awr175.

57. Sharp DJ, Scott G, Leech R. Network dysfunction after traumatic brain injury. Nat Rev Neurol. 2014;10:156-66. https://doi.org/10.1038/nrneurol.2014.15.

58. Bonnelle V, Ham TE, Leech R, Kinnunen KM, Mehta MA, Greenwood RJ, et al. Salience network integrity predicts default mode network function after traumatic brain injury. Proc Natl Acad Sci USA. 2012;109:4690-5. https://doi.org/ 10.1073/pnas.1113455109.

59. Blake DD, Weathers FW, Nagy LM, Kaloupek DG, Gusman FD, Charney DS, et al. The development of a clinician-administered PTSD scale. J Trauma Stress. 1995;8:75-90.

60. Weathers FW, Keane TM, Davidson JR. Clinician-administered PTSD scale: a review of the first ten years of research. Depress Anxiety. 2001;13:132-56.

61. Weathers FW, Bovin MJ, Lee DJ, Sloan DM, Schnurr PP, Kaloupek DG, et al. The clinician-administered PTSD scale for DSM-5 (CAPS-5): development and initial psychometric evaluation in military veterans. Psychol Assess. 2018;30:383-95. https://doi.org/10.1037/pas0000486

62. Bartlett EA, DeLorenzo C, Sharma P, Yang J, Zhang M, Petkova $E$, et al. Pretreatment and early-treatment cortical thickness is associated with SSRI treatment response in major depressive disorder. Neuropsychopharmacology. 2018;43:2221-30. https://doi.org/10.1038/s41386-018-0122-9. 